\title{
COMPETENCIA DIGITAL DE UN TUTOR E-LEARNING: UN MODELO EMERGENTE DE BUENAS PRÁCTICAS DOCENTES EN TIC
}

\section{DIGITAL COMPETENCE OF AN E-LEARNING TUTOR: AN EMERGING MODEL OF GOOD TEACHING PRACTICES IN ICT}

\author{
Inmaculada Aznar Díaz \\ Universidad de Granada, Espanha \\ iaznar@ugr.es \\ María Pilar Cáceres Reche \\ Universidad de Granada, Espanha \\ caceres@ugr.es \\ José María Romero Rodríguez \\ Universidad de Granada, Espanha \\ romejo@ugr.es
}

RESUMEN: El presente escrito forma parte de una investigación que versa sobre la representación del conocimiento sobre buenas prácticas docentes de un tutor e-learning. La metodología de trabajo es mixta, combina tanto instrumentos cuantitativos: cuestionario cerrado tipo Likert, como cualitativos: entrevista semiestructurada. El cuestionario fue elaborado ad hoc en base a las funciones y competencias del buen tutor e-learning recogidas por Yot y Marcelo (2013), aplicándose a una muestra de 13 tutores pertenecientes al departamento de tutorización de una escuela de negocios, donde se imparten enseñanzas de educación superior. Tras el análisis de los datos, se determinó a un tutor e-learning como agente de buenas prácticas en base a las respuestas satisfactorias y los criterios establecidos, al cual posteriormente se le realizó la entrevista para poder realizar una aproximación a su competencia digital. Los datos obtenidos del cuestionario se analizaron a través del software SPSS, mientras que los de la entrevista mediante el software cualitativo Atlas.ti. Finalmente fueron representados en dos mapas conceptuales, el primero recoge los datos referentes al contacto con el alumnado y la metodología docente, y el segundo muestra los aspectos relacionados con la gestión de los contenidos y la resolución de dudas.

PALABRAS CLAVE: competencia digital; e-learning; buenas prácticas docentes; representación del conocimiento.

ABSTRACT: This paper is part of a research that deals with the representation of the knowledge about good teaching practices of an e-learning tutor. The work methodology is mixed, combining both quantitative instruments, closed questionnaire type Likert, and qualitative, with a semi-structured interview. The questionnaire was elaborated ad hoc on the basis of the functions and competences of the good e-learning tutor collected by Yot and Marcelo (2013), being applied to a sample of 13 tutors belonging to the tutoring department of a business school, where higher education is taught. After the analysis of the quantitative data, an e-learning tutor was determined as an agent of good practices on 
the basis of the satisfactory answers and the established criteria, to which the interview was subsequently carried out in order to be able to make an approach to its digital competence. The data obtained from the questionnaire were analyzed through the SPSS software, while those from the interview were analyzed through the Atlas.ti qualitative software. Finally, they were represented in two concept maps: the first one gathers the data referring to the contact with the students and the teaching methodology, and the second one shows the aspects related to the management of the contents and answer for questions.

KEYWORDS: digital competence; eLearning; good teaching practices; knowledge representation.

\section{Introducción}

La tecnología ha cambiado por completo la actividad formadora, acaparando nuevas posibilidades y ámbitos que prescinden de un espacio físico y un horario establecido. La modalidad e-learning se ha consolidado como una alternativa a la formación presencial que cada vez tiene más adeptos, sobre todo por la posibilidad de compaginar la vida personal y profesional con ella.

En este trabajo se ha indagado sobre la competencia digital de un tutor e-learning para generar un modelo de buenas prácticas docentes en formación e-learning. Partiendo de este objetivo, en el siguiente escrito se recogen las fases llevadas a cabo, comenzando por la fundamentación teórica en la que se basa la investigación, el marco empírico que recoge todos aquellos aspectos relativos al proceso de investigación, los resultados y análisis de datos y por último, las conclusiones y consideraciones finales.

Cabe resaltar que se presenta un caso concreto, centrado en una escuela de negocios de la provincia de Granada de donde se ha extraído la muestra objeto de estudio. Esta institución es reconocida a nivel nacional y ha colaborado en la realización e impartición de acciones formativas e-learning con el Gobierno de España.

Por otro lado, con este proyecto se pretende invitar al lector a conocer un modelo emergente de buenas prácticas docentes a partir de la representación del conocimiento de un tutor e-learning, donde se pone de manifiesto su competencia digital.

Respecto a la metodología utilizada en este trabajo recurre a métodos mixtos, puesto que se ha empleado para la recogida de datos tanto el cuestionario cerrado tipo Likert, como la entrevista semiestructurada en función de su pertinencia para cada momento.

\subsection{Antecedentes teóricos e investigaciones previas}

Los antecedentes en los que se basa la investigación son tanto teóricos como de campo, respecto a los primeros destacan las aportaciones de Novak y Gowin (1985), Novak (1998) y González (2008) sobre el uso del mapa conceptual para la captura y representación del conocimiento, lo cual nos indica que su uso es pertinente para tal fin. Por otro lado, Nonaka y Takeuchi (1995) inciden en que el conocimiento experto es un 
conocimiento tácito, el cual presenta dificultades para su transmisión. Por ello, la presente investigación recoge las contribuciones de estos autores para emplear el mapa conceptual en el análisis del conocimiento experto para convertirlo en conocimiento explícito y dilucidar un modelo de buenas prácticas.

En cuanto a los antecedentes de campo podemos destacar el proyecto de investigación desarrollado en 6 universidades españolas sobre "Elicitación y representación del conocimiento de profesores universitarios protagonistas de buenas prácticas docentes: ingeniería del conocimiento para la mejora de la calidad de la docencia universitaria en el marco del proceso de convergencia europeo", perteneciente al Plan nacional de investigación científica, desarrollo e innovación tecnológica 2004-2007 (ARELLANO; SANTOYO, 2009; GONZÁLEZ et al. 2010; FERNÁNDEZ; MAIQUES; ÁBALOS, 2012; GONZÁLEZ; FIZ; AYERDI, 2012). Este proyecto de investigación planteó como objetivo identificar, analizar y explicitar el conocimiento experto del profesorado, agente de buenas prácticas, a través del mapa conceptual.

La muestra estuvo constituida por 136 profesores caracterizados por sus buenas prácticas docentes. Para ello, se utilizó como instrumentos de recogida de datos la entrevista en profundidad y grabaciones de sesiones de clase, de los cuales se extrajo la información para la elaboración de los mapas conceptuales. Como resultados se obtuvieron varios mapas conceptuales: uno referente a los datos biográficos del profesor o profesora; otro sobre la planificación e implementación de las materias; y un último que recoge el modo de evaluación del docente. Para finalizar todos concluyen con el uso pertinente del mapa conceptual para la representación del conocimiento experto de docentes agentes de buenas prácticas. En este sentido, la investigación desarrollada sigue la línea de este proyecto de investigación pero a diferencia de él se pone el foco de atención en las buenas prácticas de un tutor e-learning perteneciente a una institución donde se imparten estudios de educación superior.

Por otro lado, el tema sobre la competencia digital docente (CDD) está tomando un gran impulso por parte de organismos oficiales que amparan y demandan el desarrollo de habilidades digitales en la sociedad del siglo XXI en la que estamos inmersos. De modo que la tendencia sobre la implementación y el foco de interés sobre competencia digital queda reflejada en documentos como los estándares de competencia en TIC para docentes de la UNESCO (UNESCO, 2016) y de la International Society for Technology in Education de Estados Unidos (ISTE, 2017). Además, de forma más detallada Redecker y Punie (2017) recogen varios niveles en relación al grado de competencia digital que recuerdan a los mismos establecido por el Marco Común Europeo de Referencia para las Lenguas (MECD, 2012), éstos son: A1 (recién llegado), A2 (explorador), B1 (integrador), B2 (experto), C1 (líder) y C2 (pionero). En caso de impartir formación e-learning, entendemos que el nivel adecuado de un tutor debe ser entre $\mathrm{B} 2$ y $\mathrm{C} 1$, recogiendo como características específicas de un nivel B2: adaptación de las estrategias de interacción y comunicación a una audiencia específica; y de un nivel $\mathrm{C} 1$ : evaluación, reflexión e integración de la tecnología para la mejora del aprendizaje en los procedimientos formativos para estudiantes. 


\subsection{La relevancia en la gestión del conocimiento aplicada en el ámbito educativo: características de la formación e-learning y del tutor como experto}

La preocupación por la retención del conocimiento ha ocasionado un auge por su gestión en las organizaciones debido a la rotación del personal experto, los cuales poseen una gran destreza en el desarrollo de la actividad diaria. A dicha preocupación se une la dificultad para conservar este tipo de conocimiento de carácter tácito, propio de la persona, de sus creencias y experiencia personal (FRÍAS; RODRÍGUEZ, 2012).

En el ámbito educativo, Martínez y Valbuena señalan que el conocimiento profesional del profesor:

Posee un carácter práctico (manifiesto la mayoría de veces como rutinas y guiones de acción) y se construye a partir de la integración y transformación de distintos saberes, conocimientos y concepciones (disciplinares específicos, sobre la ciencia, contextuales, pedagógicos, etc.) a partir de diversas fuentes (académicas, experienciales) (MARTÍNEZ; VALBUENA, 2013, p. 118).

La relevancia de aplicar los mapas conceptuales en la investigación sobre formación docente radica principalmente en la pertinencia de esta herramienta para capturar y representar el conocimiento (DE BENITO, LIZANA; SALINAS, 2012; HOFFMAN; BEACH, 2013; DARDER, PÉREZ; SALINAS, 2014), ya que permite transformar el conocimiento tácito en explícito (RODRÍGUEZ, 2012). Siguiendo esta línea, con el análisis y representación del conocimiento experto de los docentes, agentes de buenas prácticas, se puede contribuir a su formación inicial y perfeccionamiento, generando mapas de conocimiento que reflejen modelos efectivos de actuación docente en diferentes etapas educativas y modalidades formativas, como es el caso de las TIC.

\subsubsection{La formación e-learning}

El avance de las tecnologías ha permitido el desarrollo de nuevas modalidades de enseñanza. En este sentido, la formación e-learning se ha consolidado como una alternativa a la enseñanza tradicional (FERNÁNDEZ, ÁLVAREZ; MARIÑO, 2013; HINOJO; AZNAR; CÁCERES; ROMERO, 2019; RODRÍGUEZ-GARCÍA; AZNAR; ALONSO, 2016), puesto que prescinde de espacios y tiene un carácter atemporal.

Este tipo de enseñanza virtual posibilita un espacio interactivo entre profesoradoalumnado y entre alumnado-alumnado (LLORENTE, 2006), en el cual la revisión y evaluación por pares (alumnado-alumnado) dota de autonomía al propio usuario, haciéndolo partícipe del proceso de aprendizaje.

A diferencia de la enseñanza presencial, la modalidad on-line presenta unas particularidades específicas que inciden en el papel y el rol del docente, generando unas funciones similares aunque distintas de un docente presencial. Partiendo de que el tutor se caracteriza porque "trabaja próximo al alumno, desempeñando una función de guía durante todo su proceso de enseñanza/aprendizaje" (BLÁZQUEZ; ALONSO, 2006, p. 38), en la formación e-learning este papel se acentúa, ya que la motivación y el seguimiento son una pieza esencial para mantener activo al alumnado. Del mismo modo que el trabajo colaborativo entre el alumnado se ve favorecido con el uso de las TIC (CASILLAS; 
CABEZAS; MARTÍN, 2016; RODRÍGUEZ-JIMÉNEZ; GÓMEZ; ROMERO, 2019).

Rodríguez y Calvo (2011) distinguen dos tipos de tutor e-learning: el primer tipo únicamente interviene en la tutorización pero no se encarga de diseñar el material didáctico (reactivo); en cambio el segundo tipo de tutor diseña el material y lo tutoriza (preventivo).

En la siguiente tabla (Tabla 1 ) se especifican los roles del tutor e-learning en base a Yot y Marcelo (2013).

Tabla 1: Roles del tutor e-learning.

\begin{tabular}{ll}
\hline Roles & Definición \\
\hline Docente & $\begin{array}{l}\text { Es el encargado de guiar y proveer al alumnado de recursos } \\
\text { en su proceso de aprendizaje }\end{array}$ \\
\hline Director social & $\begin{array}{l}\text { Promueve la participación y las relaciones entre todos los } \\
\text { agentes implicados }\end{array}$ \\
\hline Gerente del programa & $\begin{array}{l}\text { Apoya al alumnado en las tareas organizativas, temporales } \\
\text { (fechas y plazos) y administrativas }\end{array}$ \\
\hline Asistente técnico & $\begin{array}{l}\text { Da soporte técnico al alumnado en relación al manejo de la } \\
\text { plataforma virtual }\end{array}$ \\
\hline \multicolumn{1}{c}{ Fuente: Elaboración propia a partir de los roles establecidos por Yot y Marcelo (2013). }
\end{tabular}

Teniendo en cuenta los diferentes roles del tutor e-learning, las funciones que desempeñan son variadas. Atendiendo a Blázquez y Alonso (2009, p. 212-214) las funciones más importantes que realizan son:

a) Función docente: diseñar materiales, dominar contenido, planificar la docencia.

b) Función de orientador: guiar, motivar, dinamizar, facilitar.

c) Función técnica: conocer las tecnologías, solucionar problemas sencillos.

En consecuencia, estas consideraciones influyen directamente en la competencia digital del docente, la cual como indican Arias, Torres y Yáñez (2014) se relaciona principalmente con:

\footnotetext{
Disponer de las habilidades y capacidades para utilizar de forma eficiente los recursos y herramientas tanto de búsqueda de información como de producción y difusión de la misma, así como para comunicarla y compartirla socialmente a través de las distintas herramientas y entornos digitales (ARIAS; TORRES; YÁÑEZ, 2014, p. 359).
}

De igual forma, estos autores destacan que no solo es suficiente poseer estas habilidades sino que es necesario saber cuándo aplicarlas, cómo hacerlo y para qué utilizarlas.

\subsubsection{El tutor e-learning como agente de buenas prácticas docentes}

El grado de experto es un reconocimiento propio de la carrera, el cual se adquiere por la experiencia y conocimiento acumulado sobre determinada temática que te capacita para desempeñar el trabajo de modo más eficaz. Yot y Marcelo (2013) señalan que un 
tutor e-learning experto:

\begin{abstract}
Ha de tener dominio suficiente de la materia sobre la que enseña, ha de desenvolverse en el entorno tecnológico con solvencia, sabiendo sacar el máximo partido didáctico a las herramientas con las que cuenta, y debe poseer los suficientes conocimientos didácticos como para planificar actividades de manera realista que faciliten el aprendizaje y reorientar las estrategias si observa que éstas no dan el resultado esperado (YOT; MARCELO, 2013, p. 307-308).
\end{abstract}

Por tanto, un tutor e-learning que adquiera este grado puede considerarse agente de buenas prácticas docentes en su ámbito, ya que su conocimiento es un modelo a seguir por sus compañeros. Siguiendo a Méndez y Delgado (2016) las buenas prácticas en integración TIC se relacionan con tres bloques: proceso de enseñanza-aprendizaje (el cual centrará nuestro modelo de buenas prácticas docentes); organización y gestión del centro e; infraestructuras TIC.

En este sentido, en la investigación realizada por Rodríguez y Calvo (2011) se resaltaron entre las cualidades más importantes del tutor la rápida contestación a los correos e intervenciones, la claridad y corrección de las respuestas, la propia motivación del docente, la planificación de las actividades de debate, atención en la resolución de problemas técnicos y el envío de material complementario al alumnado.

Por último, cabe destacar que para el reconocimiento de un tutor e-learning agente de buenas prácticas docentes Cabero, Llorente y Morales (2013) aplican como criterios de selección: consideración por parte de sus compañeros como agentes de buenas prácticas, impartir más de una asignatura en red, poseer actitudes positivas y llevar más de 2 años impartiendo este tipo de formación.

\title{
2 Método
}

Este trabajo de investigación se enmarca dentro de una metodología mixta debido al tipo de instrumentos utilizados y a la naturaleza de los objetivos. De este modo, es cuantitativa puesto que se pretende cuantificar y ponderar las funciones y competencias del buen tutor e-learning a través del cuestionario cerrado con una escala tipo Likert, con la finalidad de identificar a tutores e-learning agentes de buenas prácticas docentes, para posteriormente de modo cualitativo reflejar su conocimiento sobre la formación e-learning con la ayuda de la entrevista semiestructurada.

La utilización de esta metodología es pertinente para este proyecto, ya que se procura garantizar cierta objetividad a la hora de detectar tutores e-learning, agentes de buenas prácticas. De igual forma, el análisis de su conocimiento como experto requiere de instrumentos cualitativos que permitan profundizar en la propia subjetividad de la persona.

En relación al cuestionario se diseñó de modo online a través de Google Form debido a la serie de ventajas que conlleva, entre ellas: la información queda automáticamente recogida en una hoja de Excel y a la hora de la aplicación facilita al usuario su realización. 


\subsection{Objetivos}

La investigación tiene como objetivo general profundizar en la competencia digital de tutores e-learning para generar un modelo de buenas prácticas docentes en formación e-learning. Para ello, a través del mapa conceptual el conocimiento representado aportará a la comunidad científica un modelo de buenas prácticas en el ámbito de la docencia online. sido:

Por otro lado, los objetivos específicos que vertebran y guían la investigación han

- Identificar a tutores e-learning, agentes de buenas prácticas docentes.

- Analizar la competencia digital y el conocimiento experto de los tutores e-learning, agentes de buenas prácticas.

- Representar a partir del mapa conceptual las buenas prácticas docentes que llevan a cabo.

\subsection{Muestra}

El objeto de estudio se compuso por una escuela de negocios dedicada a la formación e-learning y a distancia en educación superior y en formación bonificada. En la elección de dicha institución se ha tenido en cuenta la accesibilidad a la misma y que su actividad se basara principalmente en la formación online. En esta línea, la muestra se seleccionó a partir de un muestreo criterial o intencional, los criterios que se establecieron para su elección fueron:

- Pertenencia al departamento de tutorización, cuyo puesto laboral sea tutor elearning.

- Disponibilidad de los participantes, se les ha invitado a participar a través de las herramientas de comunicación internas de la empresa (mensaje instantáneo) explicando los motivos de la investigación y la importancia de su colaboración.

Tras aplicar estos criterios se obtuvo el número de participantes $(n=13)$ en la primera fase, en la que se administró un cuestionario tipo Likert sobre las funciones y competencias del tutor e-learning para identificar a tutores, agentes de buenas prácticas docentes. Finalmente, para seleccionar la muestra definitiva se analizaron los datos del cuestionario y se atendieron a los criterios establecidos previamente por diversos autores (RODRÍGUEZ; CALVO, 2011, YOT; MARCELO, 2013; CABERO; LLORENTE; MORALES, 2013) sobre la consideración de buenas prácticas docentes en formación e-learning, estos criterios han sido:

- Respuestas satisfactorias en todas las variables del cuestionario, es decir, que alcanzaran un rango de puntuaciones entre "3: La mayoría de veces" y "4: Siempre" de la escala Likert. Las variables del cuestionario se basan en las funciones y competencias que debe poseer un tutor e-learning (Yot y Marcelo, 2013).

- A su vez, se ha prestado especial atención a los ítems correspondientes a las cualidades más importantes del tutor establecidas por Rodríguez y Calvo (2011): rápida contestación a los correos e intervenciones, claridad y corrección de las 
respuestas, planificación de las actividades de debate, atención en la resolución de problemas técnicos y envío de material complementario al alumnado.

- Que los compañeros lo percibieran como agente de buenas prácticas docentes en tutorización e-learning, impartir más de una asignatura en red y llevar más de 2 años impartiendo este tipo de formación (CABERO; LLORENTE; MORALES, 2013).

Así pues, se constituyó la muestra final $(n=1)$ para el análisis de su conocimiento experto y modos de actuación docentes, que indican la puesta en práctica de acciones formativas de calidad en formación e-learning, debido a las buenas prácticas docentes que las sustentan.

\subsection{Instrumentos y validación}

El cuestionario inicialmente estaba compuesto por un total de 25 cuestiones: 24 preguntas recogidas en una escala Likert con 4 niveles (4: Siempre; 3: La mayoría de veces; 2: A veces; 1: Nunca) sobre las funciones y competencias del tutor e-learning y una pregunta orientada a proponer a compañeros o compañeras agentes de buenas prácticas. También se habilitó un apartado final de carácter abierto para que los participantes pudieran realizar las observaciones que estimaran oportunas.

Los 24 ítems iniciales relativos a las funciones y competencias se basan en la investigación de Yot y Marcelo (2013), los cuales determinaron las que debería poseer el buen tutor e-learning. De este modo, en la Figura 1 se muestran los ítems establecidos por cada función y competencia. 
Los tutores dan la bienvenida al alumnado y apoyan un primer contacto entre ellos, proporcionan las primeras orientaciones $y$ resuelven las primeras dudas $y$ problemas
1. ¿Sueles dar la bienvenida al nuevo alumnado matriculado?

2. En la bienvenida, ¿especificas las orientaciones y pautas que debe seguir el alumnado?

3. ¿Resuelves los problemas que presenta el alumnado?

4. ¿Te aseguras de que el alumnado comprenda el funcionamiento técnico de la plataforma?
Los tutores propician debates ajustados a la temática de estudio apoyan las líneas de discusión iniciadas por el alumnado y de todos ellos ha de realizar una conclusión de cierre
5. ¿Propones actividades de socialización para favorecer la comunicación entre el alumnado? (ejemplo: foros, redes sociales, chat)

6. En caso de utilizar actividades de socialización, ¿propicias debates sobre una determinada temática de estudio?

7. En relación a la pregunta anterior, sobre los debates: ¿Extraes una conclusión final de las aportaciones del alumnado para cerrar el debate?
Los tutores diseñan actividades adicionales de aprendizaje, ejemplifican y velan por la actualización de contenidos

\author{
8. ¿Diseñas actividades adicionales de aprendizaje? \\ 9. ¿Sueles actualizar los contenidos del temario? \\ 10. ¿Envías material complementario al alumnado? \\ 11. ¿Sueles tener un repertorio previo de recursos complementarios? \\ 12. ¿Facilitas ejemplos y casos donde ver la aplicación de los contenidos? \\ 13. ¿Envías material complementario al alumnado aunque no lo demande? \\ 14. ¿Realizas una revisión del contenido antes de empezar a trabajar el módulo? \\ 15. ¿Dominas el temario de los cursos que impartes? \\ 16. En caso de no dominar el contenido, ¿te informas sobre él antes de comenzar el curso?
}

Los tutores atienden las consultas del alumnado sobre las actividades de aprendizaje y los contenidos y les proporciona
orientaciones durante todo el proceso de enseñanzaaprendizaje para favorecer su autonomía
17. Cuando un alumno tiene alguna duda, ¿Sueles resolver con éxito sus demandas? 18. Cuando las dudas son comunes en varios alumnos, ¿envías un mensaje general al conjunto total del alumnado resolviendo esa duda?

19. ¿Envías recordatorios sobre cuestiones pendientes o eventos próximos al alumnado?

20. ¿Contestas las dudas del alumnado en un plazo máximo de 48 horas?

21. En la comunicación con el alumnado, ¿sueles transmitir ánimos y seguridad para motivar el proceso de aprendizaje del alumnado?

22. ¿Utilizas herramientas para motivar al alumnado?

23. ¿Sueles corregir en un plazo máximo de 5 días las tareas del alumnado?

24. ¿Contactas con el alumnado si notas que su actividad en la plataforma ha disminuido?

Figura 1: Preguntas establecidas en base a las funciones y competencias de Yot y Marcelo (2013, p. 314316). En la columna izquierda se recogen las funciones y competencias y en la derecha los ítems relacionados con cada una. Fuente: elaboración propia. 
Una vez obtenidos los datos recogidos a través del cuestionario se procedió a utilizar la entrevista semiestructurada para realizar una aproximación a su competencia digital y su conocimiento experto, la elección de este instrumento viene avalada por una serie de autores (GONZÁLEZ et al. 2010; DE BENITO; LIZANA; SALINAS, 2012; RODRÍGUEZ, 2012; FERNÁNDEZ; MAIQUES; ÁBALOS, 2012; GONZÁLEZ; FIZ; AYERDI, 2012; MARTÍNEZ; VALBUENA, 2013; DARDER; PÉREZ; SALINAS, 2014).

La entrevista diseñada recoge las aportaciones del planteamiento de entrevista de Yot y Marcelo (2013), así como cuestiones derivadas del cuestionario sobre cómo realizar las funciones y competencias recogidas en las 24 preguntas. Por otro lado, toma la división de variables y algunas cuestiones del trabajo de González, Fiz y Ayerdi (2012) acerca de la representación del conocimiento experto de docentes universitarios, agentes de buenas prácticas. En un primer momento la entrevista se compuso por 42 preguntas, dividiéndose en 4 variables: I. Datos biográficos; II. Experiencia docente; III. Dimensión didáctica (planificación, docencia y evaluación); y IV. Opiniones y valoraciones generales (GONZÁLEZ; FIZ; AYERDI, 2012, p. 109). Respecto a la subdivisión de la variable "III. Dimensión didáctica", se añadió el apartado "Funciones" junto a "Planificación, Docencia y Evaluación". En este apartado se han recogido las variables correspondientes a las funciones y competencias del tutor e-learning: bienvenida; dudas; socialización; motivación; y material.

En la validación de los dos instrumentos se realizó un juicio de expertos para determinar la validez de contenido, el cual consistió en invitar a un grupo de personas para que emitieran un juicio en función del diseño y estructura de los instrumentos (CABERO; LLORENTE, 2013).

Partiendo de las consideraciones de Robles y Rojas (2015) sobre los aspectos fundamentales del juicio de expertos, en la siguiente tabla (Tabla 2) se recogen los motivos por los que se realizó, algunas características de los participantes y el modo de validación.

Tabla 2: Aspectos fundamentales del juicio de expertos.

\begin{tabular}{llll}
\hline $\begin{array}{l}\text { Objetivos de } \\
\text { validación }\end{array}$ & $\begin{array}{l}\text { la } \\
\text { instrumentos }\end{array}$ & $\begin{array}{l}\text { Validar los diferentes ítems recogidos en los } \\
\text { Expertos }\end{array}$ & $\begin{array}{l}\text { Cuatro expertos pertenecientes al ámbito de la educación } \\
\text { superior: tres especializados en tecnología educativa y uno } \\
\text { en la línea de la captura y representación del conocimiento }\end{array}$ \\
\hline Modo de validación & $\begin{array}{l}\text { Método individual mediante el cual se ha obtenido la } \\
\text { información de cada experto sin que estuvieran en contacto } \\
\text { entre ellos }\end{array}$ \\
\hline
\end{tabular}

Fuente: Elaboración propia en base a Robles y Rojas (2015).

En relación a las sugerencias de mejora de los distintos expertos, se atendió a la mayoría de propuestas, excepto prescindir del ítem referente al nombre y apellidos puesto que se necesitaba conocer a quién corresponden las puntuaciones para seleccionar a los docentes agentes de buenas prácticas. De este modo, se procedió a eliminar el ítem 13; reformular la respuesta del ítem 23 aportando una lista plegable para que puedan escoger el tiempo de corrección de las tareas del alumnado (24 horas; 48 horas; 72 horas; Más de 72 horas); reformulación del ítem 3 añadiendo el sufijo "iniciales" a problemas y del ítem 
25 otorgando un carácter más directo y personal; se acotaron los intervalos de respuesta respecto al ítem sobre los años de experiencia impartiendo docencia en modalidad elearning (Menos de 1 año; Entre 1 y 5 años; Entre 6 y 10 años; Entre 11 y 15 años; Entre 16 y 20 años; Más de 20 años); utilizado en todos los ítems el tratamiento de usted; y aplicado una división por variables que responde a la clasificación establecida en la figura 1 (I. Bienvenida y resolución de dudas; II. Actividades de socialización; III. Contenido y materiales; IV. Atención al alumnado; y V. Feedback). Finalmente, tras los cambios mencionados el número total de ítems fue de 24 (23 referentes a las funciones y competencias y un ítem abierto para proponer a compañeros agentes de buenas prácticas).

Por otro lado, se atendieron todas las propuestas de mejora de la entrevista. En este sentido, se eliminaron algunos ítems, puesto que quedaban contestados en el cuestionario. También se homogeneizaron las terminaciones verbales utilizando la segunda persona con el objetivo de dar un trato más cercano y personal. Una vez aplicadas las diferentes recomendaciones la entrevista definitiva constaba de un total de 34 preguntas.

\section{Análisis de datos y resultados}

Los datos obtenidos de los 13 cuestionarios permitieron realizar un análisis pormenorizado de las cinco variables relacionadas con su práctica diaria. Cada ítem ha sido puntuado desde 4 (siempre), 3 (la mayoría de veces), 2 (a veces), hasta 1 (nunca) mediante la escala Likert, lo que facilitó el establecimiento de las medias por ítem. Estos datos fueron analizados con el software de análisis de datos cuantitativos SPSS, versión 24. Este programa estadístico permite procesar datos de carácter numérico, una vez confeccionada manualmente la matriz de datos, donde el investigador debe codificar numéricamente cada variable.

En relación a la entrevista, se dio respuesta a la mayoría de preguntas, algunas surgieron durante su transcurso para precisar la información que se obtenía y otras se omitieron, puesto que se respondían en la contestación anterior. Por tanto, los datos recogidos a través de un registro electrónico, utilizando una grabadora, resultaron de gran interés para su análisis. Este análisis fue realizado con el software de datos cualitativos Atlas.ti, debido a que permite categorizar manualmente los descriptores y a partir de su categorización elaborar mapas de categorías. A su vez, agrupa toda la información que pertenece a una misma categoría facilitando su análisis.

Atendiendo a la media el valor máximo de las puntuaciones en cada ítem ha sido 3,92 mientras que el mínimo 1,69. Respecto a la moda y mediana se situó en un máximo de 4 y mínimo de 1 y la mayor desviación típica se corresponde con un valor de 1,26, siendo muy heterogéneo el grupo en las respuestas, mientras que la menor desviación ha sido 0,27 con unas respuestas muy homogéneas. En esta línea, los ítems con mejor puntuación son los referentes a la orientación al alumnado y resolución de dudas y los de menor puntuación los relativos a la realización de actividades de socialización como el planteamiento de debates. Por tanto, las variables mejor situadas en el grupo de tutores e-learning han sido las relacionadas con la atención al alumnado (variables I, III y IV) y las más bajas actividades de socialización y feedback (variables II y V). 
En el siguiente Gráfico 1 se muestra la media de las puntuaciones por cada ítem en relación a la escala Likert. Cabe resaltar que la variable I "Bienvenida y resolución de dudas" acoge los ítems 1, 2, 3 y 4; la variable II "Actividades de socialización" los ítems 5, 6 y 7; variable III "Contenido y materiales" ítems del 8 al 15; variable IV "Atención al alumnado" del 16 al 20; y variable V "Feedback" los ítems 21, 22 y 23. En relación al ítem 22 no tiene valor en el gráfico, puesto que no se regía por la escala Likert, se daba a escoger el plazo de resolución de dudas (24, 48, 72 o más de 72 horas).

Gráfico 1: las medias de las puntuaciones de cada ítem.

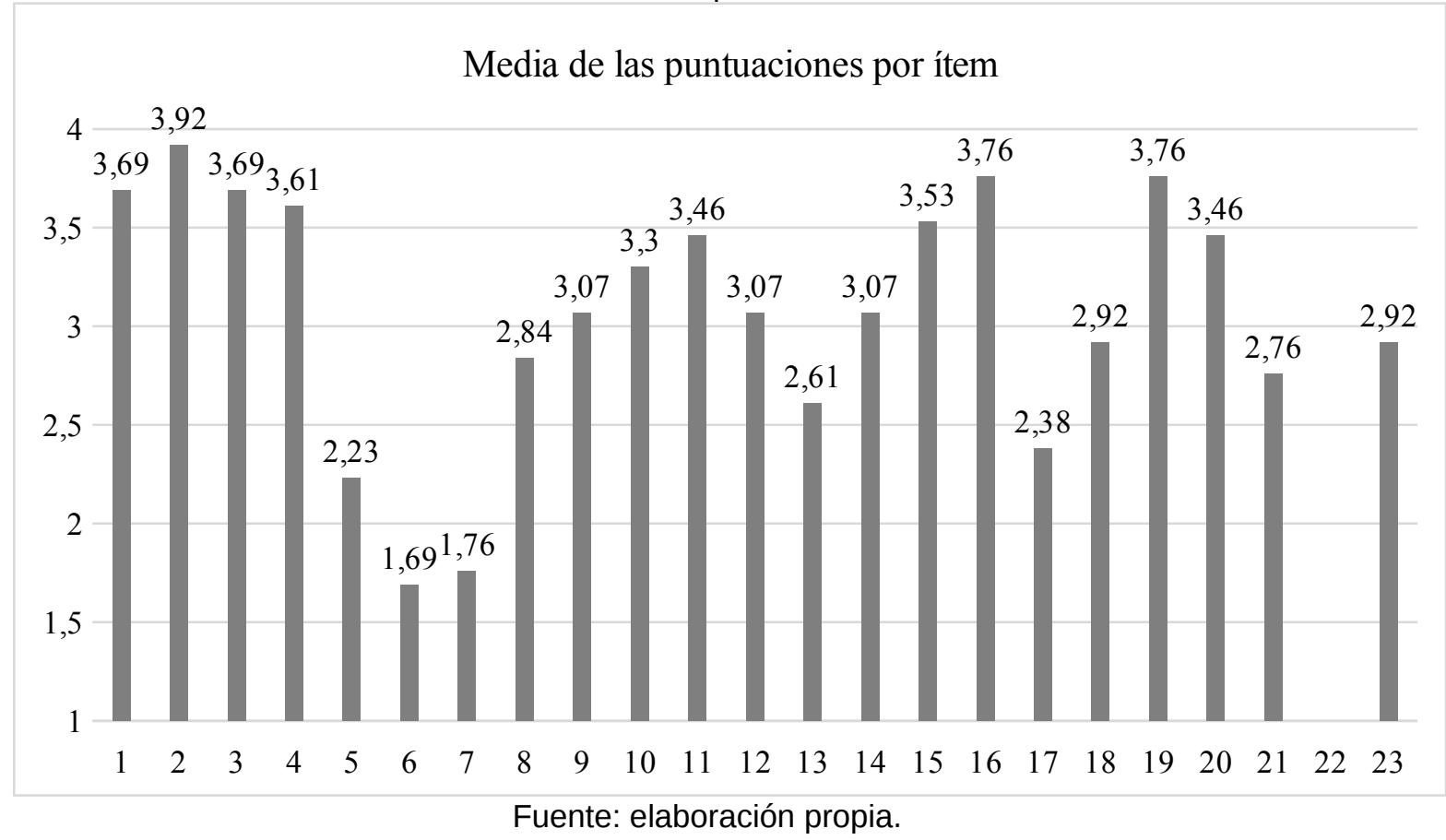

Respecto al análisis de datos de la entrevista se establecieron 10 categorías agrupadas en la familia formación e-learning (Figura 2). Las categorías responden a la división por variables de la entrevista. A partir de estas categorías se extrajo la información referente a cada una de ellas, lo que facilitó su análisis y posterior representación en dos mapas conceptuales, uno donde se recopila el contacto con el alumnado y la metodología y otro referente al contenido y resolución de dudas. Se ha optado por esta división para hacer operativa la visualización de forma gráfica de las buenas prácticas en tutorización e-learning. La graficación de los mapas conceptuales fue realizado con el software CmapTools, ya que permite su elaboración de forma y expositiva. El proceso de elaboración de los mapas conceptuales se inició con el descriptor principal, del cual se establecieron los enlaces y los demás coceptos de forma jerárquica. 


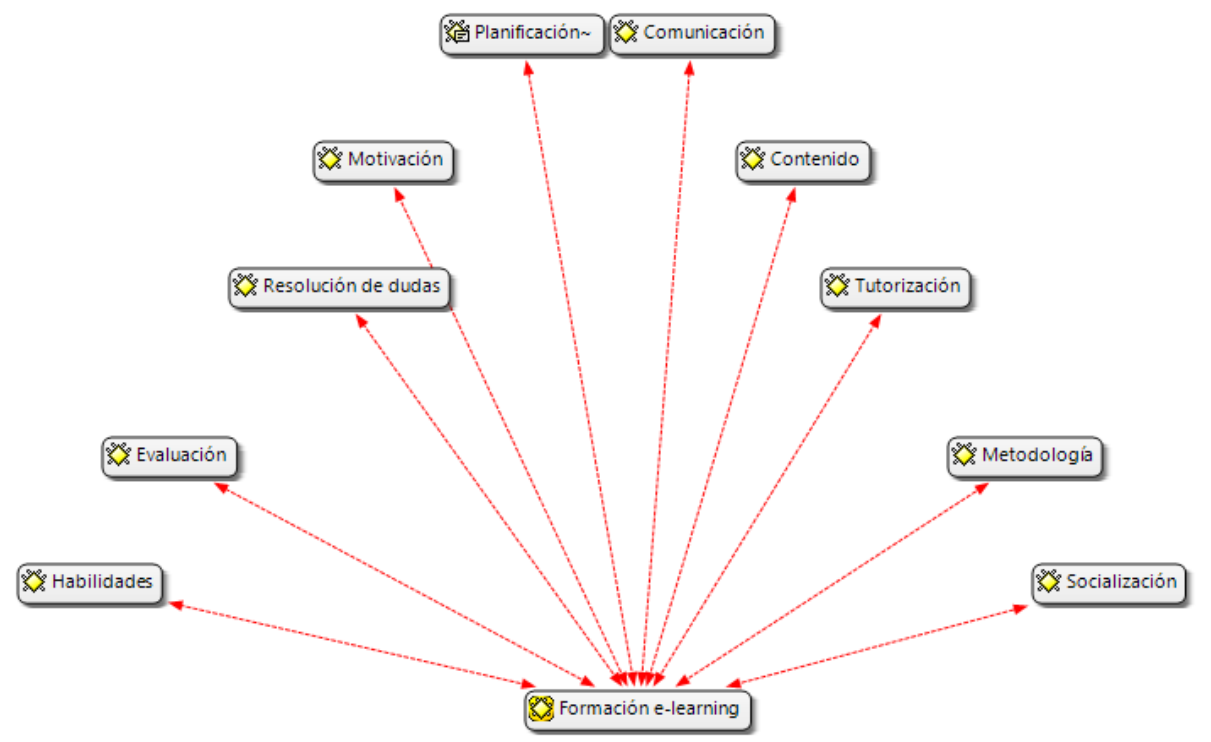

Figura 2: Familia de categorías de formación e-learning.

Fuente: elaboración propia a partir de Atlas.ti.

Los cuatro grandes conceptos en los que se organizan los dos mapas conceptuales (contacto con el alumnado; metodología individualizada; contenido; resolución de dudas) se generaron englobando las categorías previas de la familia "formación e-learning" en las que se había categorizado la información de la entrevista. De este modo las buenas prácticas docentes quedan expuestas a continuación:

En la Figura 3 se recoge de forma gráfica la información sobre todo lo que concierne a las buenas prácticas en el "contacto con el alumnado". Para ello, el contacto se realiza según la preferencia o disponibilidad, mediante tres canales principalmente: email (revisado diariamente); chat online; y llamadas telefónicas. Estas llamadas pueden ser de diferentes tipos: "bienvenida", se hacen durante los primeros días e incluyen información sobre las fechas del curso, metodología a seguir, datos de contacto, presentación del tutor para favorecer un trato cercano y cuestiones relacionadas con el material formativo; "seguimiento", para conocer el estado de la acción formativa y mantener su motivación, se llevan a cabo cada mes o dos meses; "finalización", donde se comprueba que todo está correcto para emitir el certificado; y "calidad", con la finalidad de conocer la opinión del alumnado y mejorar el contenido.

Es importante añadir que el contacto con el alumnado adquiere un interés especial si su actividad disminuye en la acción formativa, por ello se intenta poner en contacto con él para animarlo a continuar, haciendo hincapié en la necesidad inicial por la que se matriculó en el curso.

Respecto a la metodología, se aplica una "metodología individualizada", puesto que el alumnado on-line compagina la formación con la vida profesional y personal. El proceso de individualización se inicia con el primer contacto donde se conocen los motivos por los que el estudiante realiza la acción formativa. Por tanto, para el éxito de este tipo de metodología es importante contar con tres habilidades esenciales: empatía, organización y paciencia, primando el acompañamiento durante toda la acción formativa a través de los diferentes canales de comunicación.

Mientras que en la Figura 4 se engloban aspectos relativos al "contenido", el cual 
se actualiza cuando demanda el alumnado, aunque el tutor aboga por una actualización previa. El contenido con el que cuentan las acciones formativas que tutoriza es de dos tipos: obligatorio y complementario. Respecto al primero recoge un temario relativo a cada unidad, el cual es el principal aspecto evaluativo a través de pruebas tipo test, corregidas en un plazo máximo de 48 horas.

En relación al material complementario se facilita de manera individualizada en función de los intereses y grado de profundización que demande el alumnado. Proporcionando publicaciones y artículos científicos relacionados con los temas de interés del curso y proponiendo la realización de casos prácticos para realizar una intervención que recoge el proceso diagnóstico y actividades relacionadas. En ellos se evalúan las habilidades de intervención, al mismo tiempo que actúan como indicadores de comprensión del contenido.

El tutor tiene en consideración que el material que proporciona es útil y se enriquecería si se aplicara en la organización de actividades de socialización como el intercambio de temas y recursos en foros, lo que también posibilitaría una resolución de dudas entre pares.

Por otro lado, la "resolución de dudas" se realiza de forma telefónica para dudas puntuales y electrónica para dudas extensas, contestadas en un plazo máximo de 48 horas. Cuando son de carácter técnico se comprueba si puede acceder a la plataforma y si tiene presente la guía sobre cómo funciona el campus virtual. Además si las dudas son comunes en varios estudiantes el tutor envía un mensaje a todo el grupo para resolverla conjuntamente.

Como se ha mencionado, este modelo de buenas prácticas queda recogido gráficamente en dos mapas conceptuales (Figura 3 y 4).

Cabe resaltar que la experiencia como docente del tutor e-learning se basa principalmente en la formación dentro del ámbito empresarial, marcado por algunos hitos como su paso por la escuela y el aprendizaje de la modalidad e-learning a raíz de su compañera de trabajo. Por otra parte, la metodología y el trato cercano con el alumnado puede resumirse en la siguiente cita recogida en la entrevista: "yo siempre pienso que hay que ser muy positiva, yo soy muy positiva y porque creo que eso también lo transmites, si tú eres positiva el alumno lo nota y eso también le anima y le motiva a seguir su proceso de formación". 


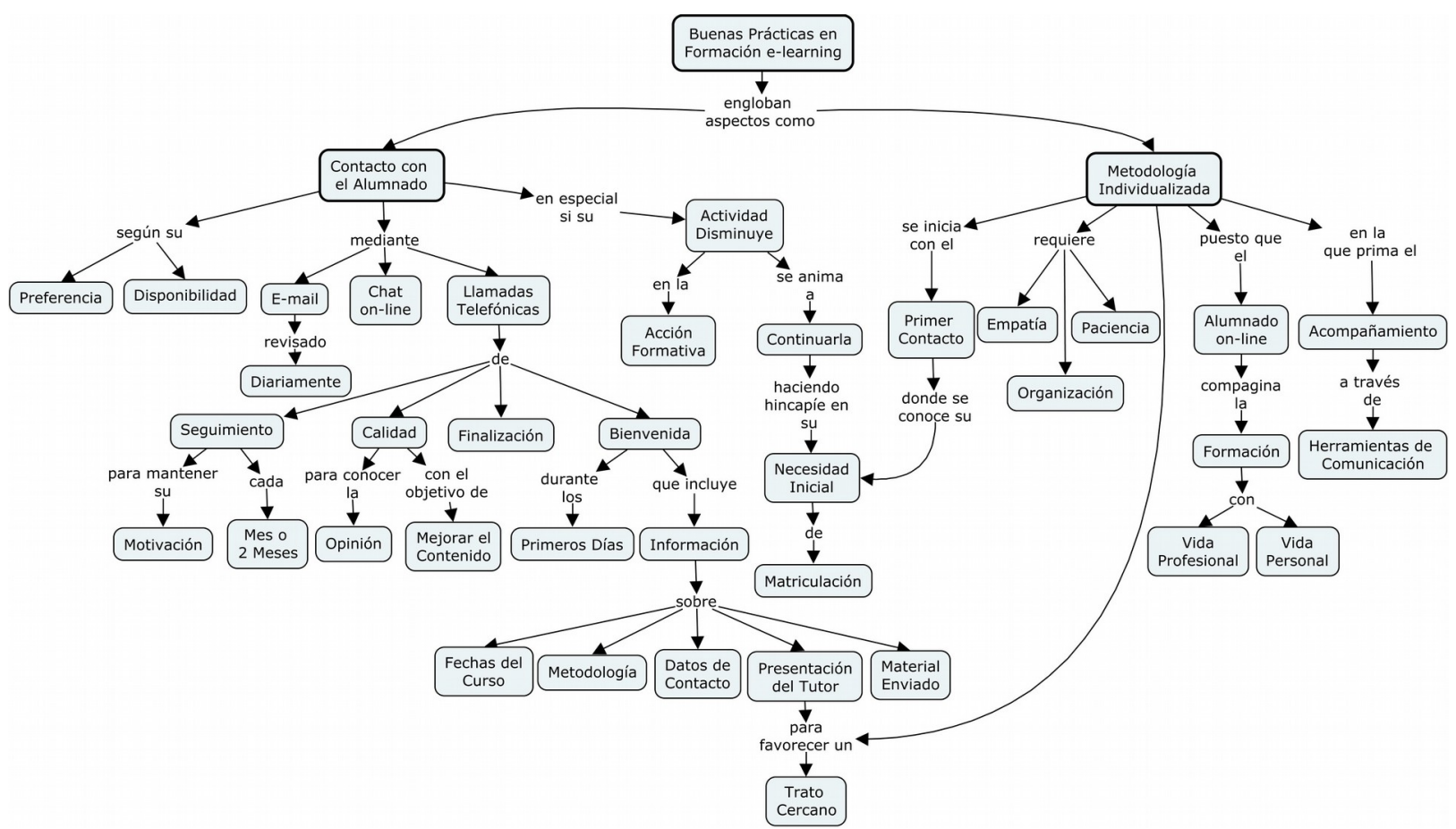

Figura 3: Mapa conceptual sobre buenas prácticas en contacto con el alumnado y tipo de metodología.

Fuente: elaboración propia a partir de CmapTools.

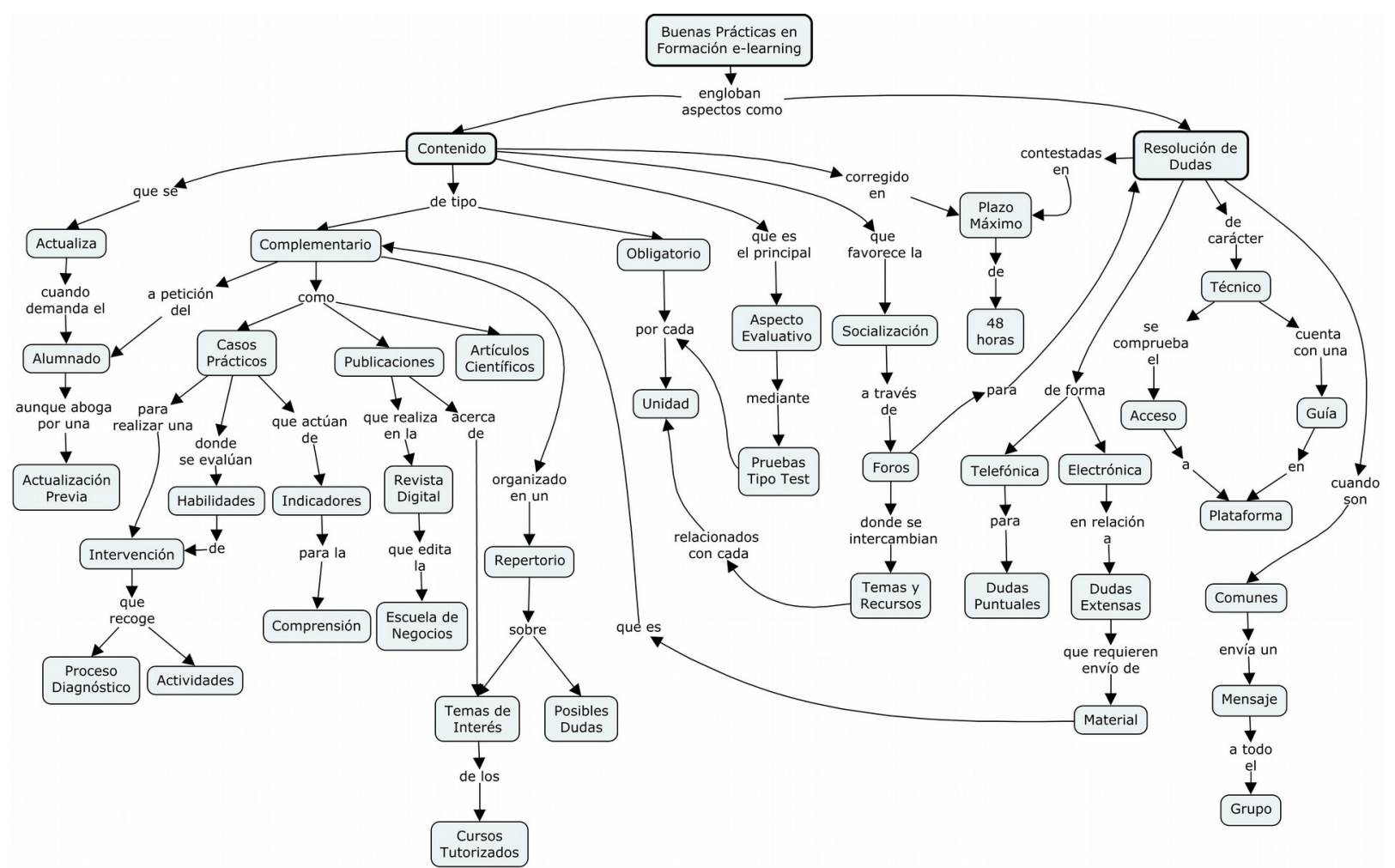

Figura 4: Mapa conceptual sobre buenas prácticas en contenido formativo y resolución de dudas del alumnado.

Fuente: elaboración propia a partir de CmapTools. 


\section{Discusión y conclusiones}

En la selección del tutor e-learning agente de buenas prácticas se tomó como principal referencia las respuestas obtenidas en el cuestionario, las cuales han estado basadas en los criterios de selección de un buen tutor e-learning establecidos por Cabero, Llorente y Morales (2013) y Yot y Marcelo (2013). Al igual, estas respuestas han sido satisfactorias respecto a las cualidades más importantes del tutor e-learning (RODRÍGUEZ; CALVO, 2011). En este sentido, la media del tutor seleccionado está por encima del grupo en relación a la rápida contestación al alumnado, preocupación por su progreso, envío de material complementario y resolución de problemas técnicos.

En cuanto a los criterios de selección para el reconocimiento de un tutor e-learning agente de buenas prácticas docentes establecidos por Cabero, Llorente y Morales (2013) se cumplen todos ellos: ha sido considerado por los compañeros como agente de buenas prácticas, imparte más de una acción formativa, posee actitudes positivas hacia la docencia y lleva más de 6 años dedicándose al sector e-learning. Por tanto, el tutor elearning que responde a las iniciales del nombre y apellidos "NBV" puede considerarse como un agente de buenas prácticas en base a las funciones y competencias del buen tutor e-learning de Yot y Marcelo (2013); las cualidades destacadas por Rodríguez y Calvo (2011); y los criterios de selección de Cabero, Llorente y Morales (2013). En consecuencia, la caracterización del tutor e-learning experto descrita por Yot y Marcelo (2013) queda constatada en el tutor entrevistado.

Como destacan Novak y Gowin (1985); González, (2008); Arellano y Santoyo (2009); De Benito, Lizana y Salinas (2012); Novak (1998; 2013); Hoffman y Beach (2013); Darder, Pérez y Salinas (2014) el mapa conceptual ha sido una herramienta válida y útil para la captura y representación del conocimiento.

Siguiendo a Rodríguez y Calvo (2011) y su distinción sobre los dos tipos de tutores e-learning, podemos afirmar que el tutor NBV responde a un tutor preventivo, el cual diseña el material y lo tutoriza.

En relación a los roles del tutor e-learning descritos por Yot y Marcelo (2013) queda constatado el rol docente, gerente del programa y asistente técnico. En cambio, el rol de director social no se ve reflejado actualmente en el puesto laboral que ocupa, ya que no hay habilitados canales de comunicación entre pares.

Por otro lado, atendiendo a las funciones más importantes del tutor e-learning establecidas por Blázquez y Alonso (2009): función docente, de orientador y técnica; todas ellas quedan reflejadas en el tutor NBV y por ende, en los mapas conceptuales. Considerando las funciones y competencias del buen tutor e-learning recogidas en Yot y Marcelo (2013) se han constatado la mayoría de ellas excepto la relativa a las actividades de socialización, debido a que en la organización carecen de ellas.

Respecto a su competencia digital docente (CDD), podemos destacar que es competente digitalmente siguiendo la aportación de Arias, Torres y Yáñez (2014), utilizando de forma eficiente los recursos y herramientas relativos a la información, la cual crea y selecciona de forma rigurosa para remitirla al alumnado. Del mismo modo que tiene el conocimiento sobre cómo hacerlo y tiene en cuenta los momentos clave para su utilización, factores que coindicen con los estándares marcados por la UNESCO (2016) e ISTE (2017). Además podría relacionarse con un grado de competencia digital de líder (C1) (REDECKER; PUNIE, 2017), teniendo en consideración que el tutor NBV utiliza de 
forma consciente y reflexiva la tecnología a su alcance para la mejora del proceso de enseñanza-aprendizaje de los estudiantes.

En definitiva, la formación e-learning tiene sus peculiaridades respecto a la formación presencial, cabe destacar que la formación en la empresa privada está influenciada por el afán económico y beneficiario, pero por ello no es menos valioso el trabajo de un tutor, puesto que la empresa privada constituye una posibilidad laboral de los profesionales de la educación. Durante el proceso de investigación se puede constatar que se ha dado respuesta a cada uno de los objetivos que se han planteado:

- Se ha identificado a un tutor e-learning, agente de buenas prácticas, utilizando el cuestionario elaborado ad hoc y validado por cuatro expertos docentes de la Universidad de Granada y especializados en formación e-learning y gestión del conocimiento.

- La entrevista semiestructurada ha permitido recoger los datos pertinentes para el análisis, mediante el software Atlas.ti, de su competencia digital y conocimiento experto como tutor e-learning, el cual ejerce su profesión en una escuela de negocios de la provincia de Granada, impartiendo acciones formativas de educación superior como máster y cursos universitarios.

- Utilizando el mapa conceptual se ha representado su experiencia, generando así un modelo emergente sobre buenas prácticas docentes en TIC.

En suma, el cumplimiento de los objetivos específicos implica la consecución del objetivo general planteado acerca de "profundizar en la competencia digital de tutores elearning para generar un modelo de buenas prácticas docentes en formación e-learning".

Finalmente, cabe resaltar como limitaciones del estudio: (i) la reducida muestra final de un solo sujeto; y (ii) no contar con las valoraciones del alumnado para la determinación del tutor agente de buenas prácticas docentes. Sin embargo, aunque solo se contó con un sujeto, el proceso seguido para la determinación de la muestra definitiva fue riguroso y mostró la realidad de la institución objeto de estudio, donde solo fue agente de buenas prácticas un tutor de un total de 13. De igual forma, la selección de este tutor fue apoyada por los comentarios de los demás tutores, por lo que se mitigó el sesgo de percepción positiva del propio sujeto. No obstante, en futuros estudios sería recomendable ampliar la muestra y contar con la opinión del alumnado.

\section{Referencias}

ARELLANO, J.; SANTOYO, M. Investigar con mapas conceptuales. Procesos metodológicos. Madrid: Narcea, 2009.

ARIAS, M.; TORRES, T.; YÁÑEZ, J. C. El desarrollo de competencias digitales en la educación superior. Historia y Comunicación Social, n. 19, p. 355-366, 2014.

BLÁZQUEZ, F.; ALONSO, L. Formación específica para tutores de e-learning. Revista Iberoamericana de Sistemas, Cibernética e Informática, v. 3, n. 1, p. 38-43, 2006.

BLÁZQUEZ, F.; ALONSO, L. Funciones del profesor de e-learning. Pixel-Bit. Revista de 
Medios y Educación, n. 34, p. 205-215, 2009.

CABERO, J.; LLORENTE, M. C. La aplicación del juicio de experto como técnica de evaluación de las tecnologías de la información (TIC). Eduweb. Revista de Tecnología de Información y Comunicación en Educación, v. 7, n. 2, p. 11-22, 2013.

CABERO, J.; LLORENTE, M. C.; MORALES, J. A. Aportaciones al e-learning desde un estudio de buenas prácticas en las universidades andaluzas. Revista de Universidad y Sociedad del Conocimiento, v. 10, n. 1, p. 45-60, 2013. DOl:10.7238/rusc.v10i1.1159

CASILLAS, S.; CABEZAS, M.; MARTÍN, J. Gestión del conocimiento: experiencias de trabajo colaborativo con estudiantes mediante Tecnologías de la Información y la Comunicación. Digital Education Review, n. 30, p. 184-206, 2016.

DARDER, A.; PÉREZ, A.; SALINAS, J. Análisis de usos del mapa conceptual en la investigación. En: CORREIA, PR; MALACHIAS, ME; CAÑAS, AJ; NOVAK, JD (ed.). Proceedings of the Sixth International Conference on Concept Mapping: Vol. 1, Concept Mapping to Learn and Innovate. Santos, Brasil: Universidad de São Paulo, 2014. p. 31-36.

DE BENITO, B.; LIZANA, A.; SALINAS, J. Los mapas conceptuales en la captura y representación de conocimiento experto docente en el uso de las TIC. En: CAÑAS, A. J.; NOWAK, J. D.; VANHEAR, J. (ed.). Proceedings of the Fifth International Conference on Concept Mapping: Vol. 3, Concept Maps: Theory, Methodology, Technology. Malta: Universidad de Malta, 2012. p. 146-149.

FERNÁNDEZ, A.; MAIQUES, J. M.; ÁBALOS, A. Las buenas prácticas docentes de los profesores universitarios: estudio de casos. Revista de Docencia Universitaria, v. 10, n. 1, p. 43-66, 2012.

FERNÁNDEZ, M. D.; ÁLVAREZ, Q.; MARIÑO, R. E-learning: otra manera de enseñar y aprender en una universidad tradicionalmente presencial. Estudio de caso particular. Profesorado, v. 17, n. 3, p. 273-291, 2013.

FRÍAS, R.; RODRÍGUEZ, C. A. Una interpretación del concepto de gestión del conocimiento de Nonaka y Takeuchi usando la ficción literaria. Apuntes del CENES, v. 31, n. 54, p. 227-260, 2012.

GONZÁLEZ, F. El mapa conceptual y el Diagrama V. Recursos para la Enseñanza Superior en el Siglo XXI. Madrid: Narcea, 2008.

GONZÁLEZ, F.; FIZ, M. R.; AYERDI, P. Un ejemplo de profesor agente de buenas prácticas docentes en el ámbito de la Sociología. Revista de Docencia Universitaria, v. 10, n. 1, p. 105-122, 2012.

GONZÁleZ, F.; GURUCEAGA, A.; POZUETA, E.; PORTA, S. Una aproximación al conocimiento de una profesora universitaria, agente de buenas prácticas docentes, utilizando mapas conceptuales. Profesorado, v. 14, n. 3, p. 117-130, 2010. 
HINOJO, F. J.; AZNAR, I.; CÁCERES, M.P.; ROMERO, J. M. Opinión de futuros equipos docentes de educación primaria sobre la implementación del mobile learning en el aula. Revista electrónica Educare, vol. 23, n. 3, p. 1-17, 2019. DOI:10.15359/ree.23-3.14

HOFFMAN, R.; BEACH, J. Lessons learned across a decade of knowledge modeling. Journal for Educators, Teachers and Trainers, v. 4, n. 1, p. 85-95, 2013.

INTERNATIONAL SOCIETY FOR TECHNOLOGY IN EDUCATION (ISTE). Estándares para la Educación en Tecnologías de Información y Comunicación (TIC), 2017. Recuperado de http://www.iste.org/standards/for-educators

LLORENTE, M. C. El tutor en E-learning: aspectos a tener en cuenta. Edutec. Revista Electrónica de Tecnología Educativa, n. 20, p. 1-24, 2006.

MARTÍNEZ, C. A.; VALBUENA, E. O. Complejidad del conocimiento profesional de una profesora de ciencias de Primaria sobre el conocimiento escolar. Campo Abierto, v. 32, n. 1, p. 117-135, 2013.

MECD. Marco Común Europeo de Referencia para las Lenguas: aprendizaje, enseñanza, evaluación. Madrid, España: Ministerio de Educación, Cultura y Deporte, 2002.

MÉNDEZ, J. M.; DELGADO, M. Las TIC en centros de Educación Primaria y Secundaria de Andalucía. Un estudio de casos a partir de buenas prácticas. Digital Education Review, n. 29, p. 134-165, 2016.

NONAKA, I.; TAKEUCHI, H. The knowledge-creating company: How Japanese companies create the dynamics of innovation. New York, NY: Oxford University Press, 1995.

NOVAK, J. D. Empowering Learners and Educators. Journal for Educators, Teachers and Trainers, v. 4, n. 1, p. 14-24, 2013.

NOVAK, J. D. Learning, Creating, and Using Knowledge: Concept Maps as Facilitative Tools in Schools and Corporations. Mahwah: Lawrence Erlbaum Associates, 1998.

NOVAK, J. D.; GOWIN, D. B. Learning How to Learn. New York, NY: Cambridge University Press, 1985.

REDECKER, C.; PUNIE, Y. Digital Competence Framework for Educators (DigCompEdu). Brussels: European Union, 2017.

ROBLES, P.; ROJAS, M. C. La validación por juicio de expertos: dos investigaciones cualitativas en Lingüística aplicada. Revista Nebrija de Lingüística Aplicada, n. 18, p. 1-16, 2015.

RODRíGUEZ, C.; CALVO, A. La figura del tutor de e-learning. Aportaciones de una investigación con estudios de caso. Revista de Universidad y Sociedad del Conocimiento, 
v. 8, n. 1, p. 66-79, 2011.

RODRÍGUEZ, S. Utilizando CMAPS para analizar el conocimiento declarado y en la acción de los asesores de formación de profesores. Journal for Educators, Teachers and Trainers, n. 3, p. 49-59, 2012.

RODRÍGUEZ-GARCÍA, A. M.; AZNAR, I.; ALONSO, S. El uso de dispositivos móviles en la práctica docente universitaria. En: BERNAL, J. L (coord.). Globalización y organizaciones educativas, XIV Congreso interuniversitario de organización de instituciones educativas. Libro de actas. Zaragoza: Universidad de Zaragoza, p. 511-518, 2016.

RODRÍGUEZ-JIMÉNEZ, C.; GÓMEZ, G.; ROMERO, J. M. Los retos tecnológicos del docente del siglo XXI. Educación y Sociedad, vol. 17, n. 2, p. 1-14, 2019.

UNESCO. Competencias y estándares TIC desde la dimensión pedagógica: una perspectiva desde los niveles de apropiación de las TIC en la práctica educativa docente. Colombia: Universidad Pontificia Javeriana, 2016.

YOT, C.; MARCELO C. Tareas y competencias del tutor online. Profesorado, v. 17, n. 2, p. 305-325, 2013.

Recebido em dia 19 de setembro de 2019. Aprovado em dia 17 de outubro de 2019. 